\title{
Papel de la nefrectomía en el adenocarcinoma renal metastático: experiencia del Servicio de Urología del Hospital Juan Canalejo
}

\author{
A. Blanco Díez, E. Fernández Rosado, G. Suárez Pascual, I. Rodríguez Gómez, \\ M.L. Ruibal Moldes, S. Novás Castro, F. Gómez Veiga, L. Álvarez Castelo, A. Barbagelata López, \\ J. Ponce Díaz-Reixa, M. González Martín
}

Servicio de Urología. Complejo Hospitalario Universitario Juan Canalejo. La Coruña.

Actas Urol Esp 2005; 29 (2): 190-197

\section{RESUMEN}

PAPEL DE LA NEFRECTOMÍA EN EL ADENOCARCINOMA RENAL METASTÁTICO: EXPERIENCIA DEL SERVICIO DE UROLOGÍA DEL HOSPITAL JUAN CANALEJO

Objetivos: Presentar nuestra experiencia en la realización de nefrectomía en tumores renales que se presentan con metástasis al diagnóstico, y observar las complicaciones, evolución y supervivencia de estos pacientes.

Material y métodos: Realizamos una revisión retrospectiva de los adenocarcinomas renales en el período entre 1-11991 y 31-12-2002. Estudiamos solamente los que se presentaron con metástasis (31). Se estudian aquellos pacientes a los que se les practicó nefrectomía y aquellos a los que no se les ofreció tratamiento quirúrgico. Buscamos mostrar las diferencias en los dos grupos en cuanto a status vital (Tabla E.C.O.G.), patología concomitante y supervivencia media. En el grupo de los pacientes nefrectomizados estudiamos las complicaciones derivadas de la intervención y el tratamiento posterior.

Resultados: Realizamos nefrectomía en 19 casos. Todos ellos E.C.O.G. O-1. La estancia post operatoria media fue de 12 días, y la tasa de complicaciones $11,5 \%$. El $45 \%$ de estos pacientes siguieron algún tipo de tratamiento posterior, y la supervivencia media fue de 31 meses. No se realizó nefrectomía en 12 casos, de los cuales 9 eran E.C.O. G 2-3. La patología asociada que presentaban estos pacientes era más importante que en el primer grupo. Sólo en 3 casos se administró tratamiento con fines paliativos y la supervivencia media fue de 3,8 meses.

Conclusiones: En pacientes con buen estado vital la nefrectomía no representa más morbilidad ni mortalidad que en los pacientes sin metástasis, y nos parece una opción fundamental en su manejo. Realizamos una revisión bibliográfica en la que recogemos las últimas tendencias en el tratamiento de estos pacientes, que ponen de manifiesto la necesidad del abordaje combinado quirúrgico e inmunoterápico. Nos parecen de interés fundamental las conclusiones de los estudios del SWOG y del EORTC.

Palabras clave: Cáncer renal. Metástasis. Nefrectomía. Cirugía.

\section{ABSTRACT}

ROLE OF NEPHRECTOMY IN METASTATIC RENAL CELL CARCINOMA. EXPERIENCE OF THE DEPARTMENT OF UROLOGY JUAN CANALEJO HOSPITAL

Objectives: We expose our experience in nephrectomy in metastatic renal cell carcinoma, and also show complications, evolution and survival of these patients.

Material and methods: We performe a retrospective review of renal cell carcinomaes treated at our service in the period between January $1^{\text {st }} 1991$ and December $31^{\text {st }} 2002$. We only studied those which presented in a metastatic pattern (31), and divide these in two groups: the ones which were nephrectomized and those which were not. We try to showw the diferences between the two groups in order of status performance (E.C.O.G.), associated morbidity and median survival. In the first group we also study complications of surgery and treatment that patients underwent.

Results: we performed nephrectomy in 19 cases, all of them E.C.O.G. 0-1. Median postoperative stay was 12 days, and complication rate was $11.5 \%$. Of these patients, $45 \%$ underwent some tipe of systemic treatment, and median survival was 31 months. We didn't performed nephrectomy in 12 patients, of which 9 were E.C.O.G. 2-3. Asociated co-morbidity was higher in this group. Only in three patients any treatment was offerted always with paliative reason. Median survival was 3.8 months.

Conclusions: In those patients with good performance status this aproach does not represent more morbility nor mortality than in non- metastatic patients, and that is a cornerstone in their management. We also make a literature review in wich we see the last pathways in the managemente of these patients, and that show the needing for a conbined aproach both quirurgical and inmunotherapical. We have review with special interest the studie's conclusions of SWOG and EORTC groups.

Keywords: Renal cancer. Metastasis. Nephrectomy. Surgery. 
$\mathrm{L}$ a nefrectomía en el cáncer de riñón cuando éste presenta metástasis en el momento del diagnóstico, es un tema controvertido. Es evidente que cualquier tumor diagnosticado en fase metastásica es de pronóstico infausto a corto plazo. Sin embargo en el adenocarcinoma renal las metástasis no llevan siempre parejo el adjetivo "terminal", debido al heterogéneo comportamiento de dicha patología, que puede comportarse de manera muy agresiva en corto período de tiempo tras la nefrectomía en pacientes no metastásicos, o lo contrario, permanecer silente durante largos períodos de tiempo en pacientes metastásicos. Esta dispar evolución unida a que la nefrectomía es una técnica quirúrgica estandarizada sin elevado número de complicaciones, y con un postoperatorio a priori incruento, hace pensar en la posibilidad de plantearles a estos pacientes dicha intervención. Las razones serían: disminuir la masa tumoral, ayudar al paciente en el ámbito psicológico sabiéndose operado, eliminar clínica asociada (sensación de pesadez abdominal, dolor, hematuria, etc.), y servir de base para un tratamiento médico de Inmunoterapia, hoy por hoy el único sistémico que ha mostrado algún efecto en alargar la supervivencia. Sin embargo, lo mismo que con cualquier intervención quirúrgica, se debe tener en cuenta el estado general del paciente, pues ésta es una alternativa agresiva de la que sólo se van a beneficiar individuos con un estado general que les permita sobrellevar un postoperatorio lo más fácil posible, y cuya evolución a medio y largo plazo, se prevea satisfactoria en lo que se refiere a causas ajenas al carcinoma renal ${ }^{1,2}$

Presentamos nuestra experiencia en el manejo de estos tumores, que aunque escasa en comparación con casuísticas americanas pensamos que es extrapolable a cualquier servicio de urología de hospital terciario de nuestro país.

\section{MATERIAL Y MÉTODOS}

Realizamos una revisión retrospectiva de los historiales clínicos de pacientes diagnosticados de carcinoma renal en nuestro Servicio entre Enero de 1991 y Diciembre de 2002.

En este período fueron diagnosticados 230 carcinomas renales, de los cuales 31 (14,3\%) tenían metástasis en el momento del diagnóstico, siendo éstos el objetivo del estudio. La edad media era 62,8 años (rango: 41-80). De ellos, 23 eran varones $(74,2 \%)$, y 8 mujeres $(25,8 \%)$. En lo referente al tumor primario, 13 (42\%) eran izquierdos, y 18 (58\%) derechos. En el estudio de Tomografía Axial Computerizada (TAC), el tamaño medio del eje mayor fue de $10 \mathrm{~cm}$. (R: 2-20), en 5 casos (16\%) existía trombo tumoral en vena cava inferior, y se observó extensión local en 3 casos (2 invasión local de hígado y 1 de mediastino y músculo psoas). Para el estudio preoperatorio de un carcinoma renal nosotros utilizamos la T.A.C. toracoabdominal o solo la abdominal asociando radiografía de tórax. En algún caso, dependiendo de la clínica o por hallazgos inespecíficos en TAC o radiografía, se utiliza la gammagrafía ósea. La TAC cerebral se realizó en aquellos casos en los que existía clínica neurológica. De los pacientes a los que se les hizo dicha prueba 2 fue por crisis comicial, 1 por accidente cerebrovascular, y 1 por parestesias, todos estos síntomas clínicos acaecidos a la par del diagnóstico del primario renal.

Estudiamos asimismo el estado general del paciente. Historia clínica para hallar patología concomitante leve o más grave (hipertensión arterial, bronquitis crónica, diabetes mellitus, antecedentes de cardiopatía isquémica, infartos de miocardio o accidentes cerebrovasculares). La exploración física nos pareció de vital importancia, así como los signos analíticos de estado general (hematocrito y creatinina plasmática). Finalmente utilizamos el Eastern Cooperative Oncology Group (E.C.O.G.) "performance status" para clasificar a cada paciente. Tabla 1 .

\section{Tabla 1}

Clasificación E.C.O.G.

E.C.O.G.

$0 \quad$ Asintomático. Totalmente activo.

1 Restricción actividad intensa. Capaz de trabajo ordinario

2 Ambulatorio y capaz de autocuidados. Incapaz para trabajar. Levantado más del 50\% del tiempo despierto.

3 Capaz de algún autocuidado. Vida cama-sillón más del 50\% del tiempo despierto.

4 Incapacidad total. Silla-cama el 100\% del tiempo despierto.

$5 \quad$ Muerto 
En nuestro estudio los pacientes fueron clasificados según la Tabla 2.

En lo que se refiere a patología concomitante asociada podemos afirmar que $18(58 \%)$ pacientes no tenían antecedentes médicos de interés, mientras que 13 (42\%) si los tenían: alcoholismo crónico, anemia severa, insuficiencia renal crónica, patología cerebrovascular, hipertensión severa, cardiopatía isquémica, antecedentes de infarto agudo de miocardio.

La distribución del E.C.O.G. en el primer grupo se observa en la Tabla 3.

La distribución del E.C.O.G en el segundo grupo fue según la Tabla 4.

Se estudian por separado dos grupos: un primero que comprende los pacientes nefrectomizados, y un segundo en el que se encuentran aquellos pacientes a los cuales no se les realizó cirugía.

Tabla 2

Clasificación E.C.O.G. de los pacientes del estudio

\begin{tabular}{lr}
\hline E.C.O.G. & \\
0 & 8 pacientes \\
1 & 14 pacientes \\
2 & 6 pacientes \\
3 & 3 pacientes \\
4 & 0 pacientes \\
5 & 0 pacientes \\
\hline
\end{tabular}

Tabla 3

E.C.O.G. de pacientes nefrectomizados

\begin{tabular}{ll} 
E.C.O.G. & \\
0 & 8 pacientes \\
1 & 8 pacientes \\
2 & 2 pacientes \\
3 & 0 pacientes \\
4 & 0 pacientes \\
5 & 0 pacientes \\
\hline
\end{tabular}

Tabla 4

E.C.O.G. de pacientes no nefrectomizados

$\begin{array}{ll}\text { E.C.O.G. } & \\ 0 & 1 \text { paciente } \\ 1 & 2 \text { pacientes } \\ 2 & 6 \text { pacientes } \\ 3 & 3 \text { pacientes } \\ 4 & 0 \text { pacientes } \\ 5 & 0 \text { pacientes }\end{array}$

Se estudian los pacientes del primer grupo atendiendo a características de cada paciente en relación a "status performance", características del tumor en lo que se refiere a tamaño y lugares de metástasis. Asimismo prestamos atención a la estancia postoperatoria y las complicaciones, el tratamiento posterior en los casos en los que se les administró, y por último la supervivencia media.

En el segundo grupo se estudian las mismas variables en lo que se refiere al paciente y al tumor, así como el tratamiento administrado, y la supervivencia.

El tiempo medio de seguimiento global fue de 19,6 meses (rango: 0,01-124). Hubo 4 pérdidas de seguimiento, 3 en pacientes no operados y 1 en paciente nefrectomizado.

\section{RESULTADOS}

\section{Pacientes nefrectomizados}

Se realizó nefrectomía en 19 casos $(61,3 \%)$. La media de edad fue de 60,7 años (rango: 44-76). Hubo 14 hombres y 5 mujeres. Todos los pacientes nefrectomizados eran E.C.O.G 0-1. En lo referente a patologías concomitantes existían 3 casos de alcoholismo crónico ( 1 con hepatopatía alcohólica) 2 de bronquitis crónica leve-moderada, y un paciente con antecedentes de linfoma de Hodking. El resto no tenía antecedentes médicos reseñables.

La técnica quirúrgica empleada fue:

- Nefrectomía radical clásica (con suprarrenalectomía)+linfadenectomía regional: 14 casos.

- Nefrectomía radical clásica (con suprarrenalectomía) sin linfadenectomía regional: 3 casos.

- Nefrectomía sin suprarrenalectomía: 2 casos.

El abordaje fue en 16 casos transperitoneal anterior, en 2 por toracofrenolaparotomía, y en 1 caso por lumbotomía.

Se realizó metastasectomía en los 2 casos en los que estaba afecta la suprarrenal contralateral. En un caso se extirpó una adenopatía cervical metastásica, y en otro se resecó una metástasis hepática única.

En lo referente al tumor, el tamaño medio en la TAC previa fue de 7,9 centímetros en su diá- 
metro mayor (rango: 2-20). Dicha cifra se correspondió con un tamaño de pieza operatoria de 8,2 centímetros (rango: 4-18,3). La anatomía patológica fue: 16 adenocarcinomas de células claras (84\%), 2 tubulopapilares (10\%), y 1 de variante sarcomatoide (6\%). Los lugares de metástasis eran:

- Cerebrales y pulmonares: 3 casos.

- Pulmonares múltiples: 5 casos.

- Pulmonares y óseas: 2 casos.

- Suprarrenales: 3 casos. 1 bilateral y 2 contralateral.

- Óseas. 3 casos.

- Hepática única. 2 caso.

- Hepática única y pulmonares: 1 caso.

La estancia post-operatoria media fue: 12 días (rango: 5-36). En 3 de estos casos (11,19 y 36 días) el retraso en el alta fue debido a complicaciones operatorias en el tercer caso, y en los dos primeros por retraso en el contacto con otras especialidades (endocrino y oncología).

- Complicaciones: 1 fístula pancreática resuelta con octreótide intravenoso que retrasó el alta hasta los 30 días. Existió asimismo un caso de infección del líquido pleural que requirió drenaje-evacuación en uno de los casos en los que se realizó un abordaje toracoabdominal, asimismo otro caso de tromboembolismo pulmonar que condición

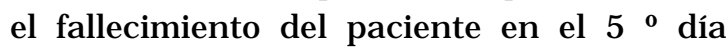
postoperatorio. La tasa de complicaciones fue de $11,5 \%$.

- 9 pacientes siguieron algún tipo de tratamiento posterior $(47 \%)$.

- Inmunoterapia con IL-2, Interferón y 5 Fluorouracilo: 4 casos.

- Inmunoterapia con IL-2, Interferón: 3 casos.

- Radioterapia: 2 casos. 1 sobre metástasis ósea y otro sobre metástasis cerebral.

Supervivencia media de este grupo de pacientes: 31 meses. (rango: 2-124).

\section{Pacientes no nefrectomizados}

No fue posible realizar nefrectomía en 12 casos $(38,7 \%)$. La media de edad de estos casos fue de 64,6 años (rango: 41-80). De ellos 9 eran hombres y 3 mujeres.

La patología asociada que presentaban estos pacientes era más importante que la que presen- taban los pacientes del grupo previo. Así tenemos 3 casos con anemia severa, (hematocrito: 18-23), 3 casos de cardiopatía isquémica con infarto agudo de miocardio, en 2 de ellos, Insuficiencia renal crónica, bronquitis crónica severa, y 2 casos de accidente cerebrovascular previo.

En lo referente al tumor el tamaño medio en la TAC previa fue de 7,2 centímetros (rango: 4-20). Los lugares de metástasis fueron:

- Hepáticas y pulmonares múltiples: 1 caso

- Hepáticas y pulmonares múltiples y suprarrenal bilateral: 1 caso

- Cerebrales y suprarrenales: 1 caso

- Suprarrenal contralateral: 2 casos

- Pulmonares: 1 caso

- Hepáticas: 3 casos

- Óseas: 1 caso

- Pulmonares, hepáticas y cerebrales: 1 caso.

- Suprarrenales y óseas: 1 caso.

Las razones para la no realización de nefrectomía en estos pacientes fueron, en unos casos la irresecabilidad de la masa, debido a tamaño o infiltración local, y en otros el número y tamaño de las metástasis. Por otra parte se tuvo en cuenta el pésimo estado general de alguno de estos pacientes y la existencia de patología asociada. Sólo 3 eran E.C.O. G 0-1. En 2 casos se realizó biopsia de la tumoración que mostró un adenocarcinoma de células claras.

En 3 casos se administró algún tratamiento (25\%): 1 radioterapia paliativa sobre el S.N.C. y en 2 casos embolización de arteria renal.

La supervivencia media fue de 3,8 meses (Rango: 0,5-6).

No se realiza análisis estadístico de los datos de supervivencia, ya que los dos grupos no son comparables. El primer grupo se nutre de pacientes con muy buen estado general y el segundo lo hace con pacientes en muy mal estado. Esto hace que no sean estudiadas diferencias en supervivencia ni las variables nefrectomía/no nefrectomía.

\section{DISCUSIÓN}

Aproximadamente 2-3\% de todos los tumores malignos del adulto se desarrollan en el riñón. En el $85 \%$ de los casos el tumor se origina en células del túbulo contorneado proximal, siendo conocidos como Tumor de Grawitz, hipernefroma o car- 
cinoma de células renales. La incidencia de dicho tumor ha crecido de manera gradual año a año pero también su tasa de curación a los 5 años que alcanza un $60 \%$ en series contemporáneas ${ }^{1}$. Sin embargo en los pacientes con enfermedad metastásica la supervivencia a 2 años oscila entre 0 y $20 \%$. En el momento del diagnóstico, $20 \%$ de los pacientes tienen enfermedad diseminada y un $25 \%$ localmente avanzada.

La cirugía es el tratamiento princeps para el carcinoma renal localizado, pero su realización en pacientes con enfermedad metastásica, siempre ha estado rodeada de polémica. Actualmente con el desarrollo de nuevas estrategias inmunoterapéuticas, ha llegado el momento de replantearnos dichas actitudes.

Existen argumentos a favor y en contra de dicha actuación Tabla 5.

Es evidente que la nefrectomía en un paciente con diseminación metastática no le va a proporcionar la curación, es más, el paciente es probable que fallezca debido a sus metástasis y no a su tumor primario. Por tanto los motivos más racionales para ofertar dicha intervención son el beneficio potencial en supervivencia, o la mejoría de la calidad de dicha supervivencia.

Clásicamente, las opciones terapéuticas en un paciente con carcinoma renal metastático y con buen estado general que le permitiesen sobrellevar una terapia agresiva eran:

1. Nefrectomía y/o metastasectomía solo.

2. Cirugía citorreductiva seguida de inmunoterapia.

3. Inmunoterapia inicial seguida de nefrectomía en respondedores.

4. Inmunoterapia solo.

Hoy en día parece que la nefrectomía por si sola no mejora la supervivencia. Las ventajas teóricas de la nefrectomía serían la eliminación de la fiebre de origen tumoral, la extracción de una fuente de secuestro de linfocitos, la prevención de complicaciones durante el tratamiento sistémico, la posibilidad de cultivar linfocitos que infiltran el tumor para usarlos en terapias experimentales, y la reducción de una masa tumoral inmunosupresora. Otras consideraciones prácticas serían la mejora del status vital, permitiendo mejor tolerancia y respuesta a tratamiento sistémico, eliminación del tumor primario, como posible fuente de metástasis, y la evidencia de la escasa respuesta a la inmunoterapia en el tumor primario.

Cuando se evalúa esta cirugía, una de las cuestiones más importantes es saber si los pacientes con enfermedad metastática tolerarán la operación como para recibir después inmunoquimioterapia, y si la extirpación del riñón aumentará las posibilidades de una respuesta objetiva a la inmunoterapia en los lugares de metástasis. Este procedimiento quirúrgico supone, en manos experimentadas, uno niveles aceptables de morbimortalidad. Sin embargo también es verdad que entre el $38 \%$ y el $77 \%$ de estos pacientes no llegarán a recibir inmunoterapia por el rápido avance de la enfermedad ${ }^{1}$.

Según todo lo expuesto, es evidente que la clave de dicho abordaje es la correcta selección de los pacientes. Teóricamente se debe limitar esta cirugía a pacientes que se van a beneficiar del abordaje combinado, y por tanto los criterios de inclusión deben ser rigurosos.

En 1978 de Kernion concluyó que la nefrectomía añadida al tratamiento standard en pacientes con enfermedad avanzada no aumentaba la supervivencia $^{2}$. Asimismo otras publicaciones ${ }^{3}$ concluyen que la cirugía radical no tiene impacto alguno en la supervivencia final de estos pacientes.

Sin embargo existen dos amplios estudios randomizados que merecen toda nuestra atención: El estudio SWOG, y el estudio de la EORTC.

\section{Tabla 5}

\section{A favor}

- Tratamiento/prevención de complicaciones (hematuria, sangrado, dolor)

- Regresión espontánea de metástasis

- Extracción de la fuente de metástasis

- Reducción de masa tumoral

- Extracción de una fuente de secuestro de linfocitos circulantes

- Mejoría psicológica o de calidad de vida

\section{En contra}

- Alternativas al tratamiento (embolización)

- Inútil (pérdida de tiempo)

- Peligrosa (morbimortalidad)

- Ausencia de pruebas de un beneficio en la supervivencia

- Puede comprometer el sistema inmune 
El Estudio SWOG (Southwest Oncology Group) comparó la nefrectomía seguida de inmunoterapia con inmunoterapia sola en 241 pacientes con carcinoma renal metastático ${ }^{4}$. Los pacientes fueron randomizados para nefrectomía radical seguida de interferón $5 \times 106 \mathrm{IU} / \mathrm{m} 2$ subcutáneo 3 veces por semana, o interferón solo. La supervivencia media fue de 11,1 meses para el primer grupo, y 8,1 meses en el segundo grupo $(p=0,05)$. Las conclusiones fueron que la nefrectomía seguida inmunoterapia debe ser el manejo standard para pacientes con carcinoma renal metastático.

El estudio de la EORTC (Genitourinary Group of the European Organisation for Research and Treatment of Cancer) confirmó los datos del SWOG. Se randomizaron 85 pacientes para los mismos grupos de tratamiento y a las mismas dosis que el estudio previo. Los pacientes del primer grupo tenían un tiempo medio hasta progresión significativamente más amplio que los del segundo grupo (5 vs 3 meses, p=0,04), así como mayor supervivencia ( 17 vs 7 meses, $p=0,03$ ). Los investigadores también concluyeron que la nefrectomía previa a la inmunoterapia se debía recomendar como tratamiento standard para determinados pacientes ${ }^{5}$.

El resultado de un meta-análisis de datos de los dos estudios se presentó en 2002 por R. Flanigan, y vino a confirmar la ventaja de la combinación, con un incremento en la mediana de supervivencia de 5 meses. Dicho autor va más allá expresando que la supervivencia de estos pacientes puede ser aún mayor cuando se aplican los regímenes más agresivos de inmunoterapia basada en Interleukina 2. Expone asimismo que los pacientes que más se benefician de esta doble terapia son aquellos con buen estatus vital, metástasis sólo pulmonares y una adecuada reserva cardiopulmonar ${ }^{6}$. El mismo grupo en un trabajo previo expone diferencias significativas de supervivencia entre pacientes tratados con Interferón alfa $2 \mathrm{~b}$, solamente, y aquellos pacientes tratados con Interferón alfa $2 \mathrm{~b}$ asociado a nefrectomía previa, independientemente del estatus vital, lugares de metástasis, y presencia o ausencia de lesión metastásica medible ${ }^{7}$.

Un grupo muy activo en el estudio de estos pacientes es el de UCLA. En tres trabajos dife- rentes, muestran la evidencia de un abordaje agresivo combinado (nefrectomía e inmunoterapia), en el manejo de esta entidad. El 10\% de estos pacientes requirieron resección concomitante de un trombo en la cava, $5 \%$ hepatectomía parcial, 3\% cirugía duodenal, y 1 (2\%), esplenectomía. Los datos de evolución postoperatoria no son diferentes a los de la cirugia en pacientes sin metástasis y concuerdan con los nuestros (12\% de complicaciones), sin mortalidad postoperatoria $^{8}$. En otro estudio el UCLA Medical Center Kidney Cancer Program estudia 354 pacientes consecutivos con carcinoma renal metastático, dividiéndolo en cinco grupos y estudiando las diferencias en supervivencia entre los grupos

Grupo 1: Interleukina 2 sin resección de tumor primario

Grupo 2: Nefrectomía seguida de Interleukina 2,

Grupo 3: Nefrectomía seguida Interleukina 2 y linfocitos infiltrantes de tumor. Los pacientes a los que se les realizó nefrectomía por tumor localizado se dividieron en otros dos grupos.

Grupo 1: Los que desarrollaron metástasis a partir de los primeros 6 meses, a los que se les administró Interleukina 2.

Grupo 2: Los que desarrollaron metástasis en los primeros seis meses y se les administró Interleukina 2.

La mejor tasa de supervivencia se observó en el Grupo 3. Estas cifras muestran que la cirugía agresiva es segura, con mínima morbimortalidad independientemente del tamaño tumoral. Asimismo, mejora significativamente la supervivencia de estos pacientes cuando se asocia a un régimen de inmunoterapia ${ }^{8-10}$. Otro grupo californiano (University of California, San Francisco), presenta resultados similares ${ }^{11,12}$. De 63 pacientes estudiados sólo la mitad tenían metástasis en un solo órgano, y que en el $27 \%$ de estos pacientes fue necesaria la cavotomía para extraer el tumor en su totalidad. Sólo $62 \%$ siguieron un tratamiento sistémico posterior. La media de supervivencia fue de 17,8 meses $^{12}$. La conclusión es la misma: en el contexto de un tratamiento sistémico del carcinoma renal, la nefrectomía representa un papel central.

En diferentes términos ofrece un trabajo el grupo de Novick de la Cleveland Clinic, Ohio. Se pretende establecer el papel de la nefrectomía 
adyuvante en este tipo de enfermos. Se estudiaron 62 pacientes con carcinoma renal metastático. Se dividieron en dos grupos: 37 pacientes siguieron el esquema de nefrectomía seguida de inmunoterapia y 25 pacientes inmunoterapia seguida de nefrectomía en aquellos pacientes respondedores. En el primer grupo, $22 \%$ de pacientes no pudieron complementar el tratamiento con modificadores de respuesta biológica debido a complicaciones perioperatorias, contraindicaciones médicas, progresión tumoral, o muerte. El $8 \%$ tuvo una respuesta parcial y la supervivencia media fue de 12 meses. En el segundo grupo 3 (12\% de pacientes) tuvieron respuesta objetiva ( 2 completa y 1 parcial), con una supervivencia media de 14 meses. Estos resultados suponen para los autores la confirmación del papel de la nefrectomía adyuvante en el tratamiento de estos pacientes ${ }^{13}$.

Todos estos resultados son concluyentes ${ }^{14,15}$ pero al igual que observamos en nuestros propios resultados, no todos los pacientes a los que se les realiza nefrectomía con la intención de administrarles un tratamiento complementario posterior, recibirán dicho tratamiento, bien por complicaciones postnefrectomía, bien por progresión, o por otras causas. En nuestra experiencia sólo 7 de los 19 pacientes (37\%) siguieron un tratamiento sistémico. ¿Realmente tiene un papel la nefrectomía en estos casos en los que después no se complementará con nada?. Nosotros pensamos que si, ya que la morbimortalidad de esta operación la consideramos aceptable, por lo que debe ser incluida en el protocolo de todos los pacientes con cáncer renal metastático y buen estatus vital. Existen estudios en los que sin tener en cuenta el tratamiento posterior se demuestra la mayor supervivencia de pacientes a los que se les ha realizado esta cirugía reducto$\mathrm{ra}^{16}$. En este sentido merece nuestra atención un trabajo recientemente publicado por el Departamento de Urología de la Universidad de Indiana, en el cual se presenta la evolución y supervivencia de 32 pacientes con cáncer renal metastático y pobre estatus vital a los que se les realizó nefrectomía. La media de estancia hospitalaria fue de 5.1 días. Solo hubo 6 complicaciones postoperatorias, y lo que es más importante, el $73 \%$ de los 29 pacientes valorables a las cua- tro semanas, presentaban un status igual o mejor que preoperatoriamente, incluyendo 4 pacientes que pasaron de un E.C.O.G.: 2, a 0 ó 1. Un $34 \%$ de estos pacientes con pobre estado general pudieron tras la cirugía seguir un régimen de inmunoterapia. Por tanto la vista de estos resultados nos demuestra de nuevo que la nefrectomía radical en el carcinoma renal metastático tiene baja morbimortalidad y aceptable evolución incluso en pacientes con pobre estatus vital $^{17}$.

Clásicamente se exponía la cirugía en estos pacientes como posibilitadora de la regresión de las metástasis sobre todo las pulmonares y únicas. Hoy en día es evidente que dicho comportamiento del tumor es poco frecuente y que no justificaría por si solo la cirugía ${ }^{18,19}$.

Por último una reflexión: el 30\% de los pacientes con adenocarcinoma renal se presentan con metástasis al diagnóstico que podamos detectar en pruebas de imagen, pero otros $30 \%$ tendrian metástasis subclínicas que no podemos detectar, y que sin embargo se operan sin controversias. ¿Qué haríamos con este último grupo de pacientes?, ¿no operarlos?. La respuesta es evidente: los operaríamos. ¿Por qué dudarlo cuando las detectamos en pruebas de imagen? ${ }^{20,21}$.

Como conclusiones: en un paciente con cáncer renal y metástasis al diagnóstico, el manejo consiste en nefrectomía, con las mismas contraindicaciones de estado general que se impondrían en paciente sin metástasis, y posteriormente tratamiento con Inmunoterapia. Los resultados son pobres, pero halagüeños para el tratamiento de estos pacientes ${ }^{20,21}$.

\section{CONCLUSIONES}

En nuestra serie, la nefrectomía radical no parece aumentar el índice de complicaciones, realizada en este tipo de pacientes, en comparación con individuos sin enfermedad metastásica, siempre en cuando sea adecuada la selección de los mismos. Por otra parte la supervivencia de dichos pacientes nefrectomizados nos parece superior a los pacientes a los que no se realiza cirugía, sin clínica derivada de su masa tumoral primaria. Nosotros pensamos que la nefrectomía en presencia de metástasis podría tener importante papel paliativo, psicológico, y de mejoría clínica. 
Asimismo creemos que se debería indicar a los pacientes que vayan a recibir inmunoquimioterapia, como lo muestran los estudios expuestos. Hoy en día con las posibilidades que se abren en el campo de la oncología con el mejor conocimiento de los moduladores de respuesta biológica para el tratamiento de estos pacientes la nefrectomía se muestra como pilar importante en el manejo inicial de los mismos. Sin embargo, como vemos en nuestra serie, de los pacientes operados sólo alrededor del 40\% complementarán su tratamiento con algún tipo de inmunoterapia, como comentamos anteriormente. Ésto es debido a progresión de enfermedad, emperoamiento de la clínica, etc., y concuerdan estos porcentajes con otras series ${ }^{1,12,13,17}$.

\section{Agradecimientos}

A todos nuestros pacientes, de los que aprendemos día a día, y que nos enseñan con humildad.

\section{REFERENCIAS}

1. Mickisch, GH. Surgery for metastatic renal cell carcinoma: a matter of attitude? Urol Clin North Am1999;51:39-43.

2. Dekernion, JB, RammingKP, Smith RB. The natural history of metastatic renal carcinoma: a computer analysis. J Urol 1978;120:148-152.

3. Bromwich E, Hendry D. Aitchison M. Cytoreductive nephrectomy: is it a realistic option in patients with renal cancer?. BJU Int 2002;89:523-525.

4. Flanigan RC, Salmon SE, Blumenstein BA, Bearman SI, Roy V, McGrath PC, Caton JR Jr, Munshi N, Crawford ED. et al. Nephrectomy followed by interferon alfa 2-b compared with interferon alfa 2.b alone for metastatic renal cell cancer. N Eng J Med 2001;345:1655-1659.

5. Mickisch GH, Garin A, Van Poppel H, De Prijck L, Sylvester R. Radical nephrectomy plus interferon-alfa-based inmunotherapy compared with interferon alfa alone in metastatic renal cell carcinoma: a randomised trial. Lancet 2001;358:948-949.

6. Flanigan RC. Cytoreductive nephrectomy in metastatic renal cancer. Curr Urol Rep 2003;4:36-40.

7. Campbell SC, Flanigan RC, Clark JI. Nephrectomy in metastatic renal cell carcinoma. Curr Treat Options Oncol 2003;4:363-372.

8. Franklin JR, Figlin R, Rauch J, Gitlitz B, Belldegrun A. Cytoreductive surgery in the management of metastatic renal cell carcinoma: the UCLA experience. Semin Urol Oncol 1996;14:230-236.
9. Belldegrun A, Shvarts O, Figlin R. Expanding the indications for surgery and adyuvant interleukin-2 based inmunotherapy in patients with advanced renal cell carcinoma. Cancer J Sci Am 2000;6 Suppl 1:88-92.

10. Han KR, Pantuck AJ, Bui MH. Number of metastatic sites rather than location dictates overall survival of patients with node-negative metastatic renal cell carcinoma. Urology 2003;61:314-319.

11. Wolf JS, Aronson FR, Small EJ, Carroll PR. Nephrectomy for metastatic renal cell carcinoma: a component of systemic treatment regimens. J Surg Oncol 1994;55:7-13.

12. Tigrani VS, Reese DM, Small EJ, Presti JC, Carroll PR. Potential role of nephrectomy in the treatment of metastatic renal cell carcinoma: a retrospective analysis. Urology 2000;55:36-40.

13. Rackley R, Novick A, Klein E, Bukowski R, Mclain D, Goldfarb D. The impact of adjuvant nephrectomy on multimodality treatment of metastatic renal cell carcinoma. J Urol 1994;152:1399-1403.

14. Cooney MM, Remick SC, Vogelzang NJ. A medical oncologis't approach to inmunotherapy for advanced renal tumors: is nephrectomy indicated?. Curr Urol Rep 2004;5:19-24.

15. Chaudhary UB, Hull GW. The evolving role of cytoreductive surgery for metastatic renal cell carcinoma. Oncology 2003;17:701-705.

16. Uygur MC, Usubutun A, Ozen H, Ayhan A, Kendi S. Prognosis factors and the role of nephrectomy in metastatic renal cell carcinoma. Int Urol Nephrol 1998;30:681687.

17. Mosharafa A, Koch M, Shalhav A. Nephrectomy for metastatic renal cell carcinoma: Indiana University experience. Urology 2003;62:636-640.

18. Sawczuk IS, Pollard JC. Renal cell carcinoma: should radical nephrectomy be performed be performed in th presence of metastatic disease? Curr Opin Urol 1999;9:377381 .

19. Vogelzang NJ, Shipley WU, Scardino PT, Coffey DS, Miles BJ. Managemente of metastasic renal cell carcinoma. Comprehensive Textbook of Genitourinary Oncology. Ed. Lippincott Williams and Williams. Philadelphia. 1999.

20. Flanigan RC, Mickisch GH, Sylvester R, Tangen C, Van Poppel H, Crawford ED. Cytoreductive nephrectomy in patients with metastatic renal cancer: a combined analysis. J Urol 2004;171:1071-1076.

21. Flanigan RC. Debulking nephrectomy in metastatic renal cancer. Clin Cancer Res 2004;10:6335-6341.

Dr. A. Blanco Díez

Calle El Reventón, 23

Monte Lentiscal

35310 Las Palmas de Gran Canaria.

(Trabajo recibido el 6 septiembre de 2004) 